JPE 11-3-8

\title{
Design of a Discrete Flux Observer by the Power Series Approximation
}

\author{
Kyung-Seo $\mathrm{Kim}^{\dagger}$ and Il-Han Kim* \\ $\dagger^{\dagger *}$ R\&D Center, LSIS, Anyang, Korea
}

\begin{abstract}
The power series approximation method is proposed for real time implementations of a discrete flux observer. The proposed method improves the performance of the discrete flux observer in the case of a low sampling rate and high speed range, where the simple discrete flux observer converted by the Euler method cannot estimate the actual flux precisely. The performance of discrete flux observers with different orders of approximation is compared to find out the proper order of approximation. The validity of the proposed method is verified through simulation and experiment.
\end{abstract}

Key Words: Discrete Time Systems, Flux Observers, Induction Motor Drives

\section{INTRODUCTION}

A flux observer is often used in industrial drive systems to estimate the rotor flux which is required for sensorless vector control or direct field-oriented control of induction motors. The performance of the system depends on the accuracy of the flux estimation because the system design is based on the assumption that the estimated flux coincides with the real motor flux.

Many research publications have focused on the performance of a flux observer at the range of very low speed because a precise estimation of the real rotor flux is quite difficult at this range [1]-[4]. This paper, on the other hand, focuses on the performance of a flux observer at the high speed range, especially with a low sampling rate.

For a digital implementation, the Euler method is a simple method to convert a continuous time observer into a discrete time observer. If the sampling rate isn't high enough, this simple flux observer cannot estimate the real flux precisely, which results in a degradation of the system performance.

The power series approximation is proposed in this paper for real time implementations of a discrete flux observer. The proposed method improves the performance of the observer with a low sampling rate and under a high speed drive range. The accuracy of the discrete time flux observer can be improved by increasing the order of the power series approximation at the cost of a high computational load. Four cases with different orders of approximation are compared to find out the proper order of approximation by considering the system performance and the computational complexity.

\footnotetext{
Manuscript received Dec. 14, 2010; revised Apr. 18, 2011

Recommended for publication by Associate Editor Jang-Mok Kim.

$\dagger$ Corresponding Author: kyungseok@1sis.biz

Tel: +82-31-450-7569, Fax: +82-31-453-6263, LSIS Co., Ltd.

* R\&D Center, LSIS, Korea
}

\section{A Simple Discrete Flux Observer}

An induction motor model can be expressed by the state equations as follows,

$$
\begin{aligned}
\frac{d}{d t}\left[\begin{array}{c}
\mathbf{i}_{s} \\
\Phi_{r}
\end{array}\right] & =\left[\begin{array}{ll}
\mathbf{A}_{11} & \mathbf{A}_{12} \\
\mathbf{A}_{21} & \mathbf{A}_{22}
\end{array}\right]\left[\begin{array}{c}
\mathbf{i}_{s} \\
\Phi_{r}
\end{array}\right]+\left[\begin{array}{c}
\mathbf{B}_{1} \\
0
\end{array}\right] \mathbf{v}_{s} \\
& =\mathbf{A} \mathbf{x}+\mathbf{B u}
\end{aligned}
$$

where,

$$
\begin{aligned}
& \mathbf{i}_{s}=\left[\begin{array}{c}
i_{d}^{s} \\
i_{q}^{s}
\end{array}\right] \quad \Phi_{r}=\left[\begin{array}{c}
\Phi_{d}^{r} \\
\Phi_{q}^{r}
\end{array}\right] \quad \mathbf{v}_{s}=\left[\begin{array}{c}
v_{d}^{s} \\
v_{q}^{s}
\end{array}\right] \\
& \mathbf{A}_{11}=-\left\{\frac{R_{s}}{\sigma L_{s}}+\frac{1-\sigma}{\sigma \tau_{r}}\right\} I \\
& \mathbf{A}_{12}=\frac{L_{m}}{\sigma L_{s} L_{r}}\left\{\frac{1}{\tau_{r}} I-\omega_{r} J\right\} \\
& \mathbf{A}_{21}=\frac{L_{m}}{\tau_{r}} I \\
& \mathbf{A}_{22}=-\frac{1}{\tau_{r}} I+\omega_{r} J \\
& \mathbf{B}_{1}=\frac{1}{\sigma L_{s}} I \\
& I=\left[\begin{array}{ll}
1 & 0 \\
0 & 1
\end{array}\right] \quad J=\left[\begin{array}{cc}
0 & -1 \\
1 & 0
\end{array}\right]
\end{aligned}
$$

$\mathbf{i}_{s}, \Phi_{r}$ and $\mathbf{v}_{s}$ are the stator current vector, the rotor flux vector and the state voltage vector respectively.

The observer for the flux estimation of induction motors can be constructed as follows,

$$
\frac{d}{d t} \hat{\mathbf{x}}=\hat{\mathbf{A}} \hat{\mathbf{x}}+\mathbf{B u}+\mathbf{G}\left(\hat{\mathbf{i}}-\mathbf{i}_{s}\right)
$$

where $\mathbf{G}$ is the observer gain matrix to compensate for the error. The " ^ " symbol denotes the estimated variables. 
To implement the flux observer (2) on a microprocessor, it is necessary to convert the continuous-time state equations into the discrete form. Even though the system matrix $\hat{\mathbf{A}}$ contains the time-varying component $\hat{\omega},(2)$ can be considered as a linear time invariant system during one sampling period because the rotor speed $\hat{\omega}$ varies slowly.

The Euler method is the simplest and most common method to convert continuous-time state equations into discrete-time state equations. The following equation is the discrete form of (1) converted by the Euler method, where $T_{s}$ is the sampling time.

$$
\frac{\mathbf{x}(n+1)-\mathbf{x}(n)}{T_{s}}=\mathbf{A x}(n)+\mathbf{B u}(n)+\mathbf{G}\left(\hat{\mathbf{i}}(n)-\mathbf{i}_{s}(n)\right)
$$

If the induction motor is driven by a PWM inverter, it is natural to coincide the sampling period of the controller with the switching period of the power switches. In commercial inverters, the switching frequency of the power switch is usually higher than $8 \mathrm{kHz}$ because this frequency value is proper considering both the audible noise and the switching loss. In the case of medium capacity drives, however, excessive heat is generated with this switching frequency. To reduce the amount of heat caused by the switching loss, the switching frequency needs to be decreased, which results in a reduction of the sampling rate. The reduction of the sampling rate causes a problem in that the simple discrete flux observer cannot estimate the real flux value correctly.

\section{Simulation I : Simple Discrete Flux Observer}

A computer simulation is performed using Matlab/ Simulink. The target machine is an induction motor rated at 1.5 $\mathrm{kW}$ and $220 \mathrm{Vrms}$. The flux regulator controls the magnitude of the estimated flux in relation to its rated value.

Fig. 1 shows the real flux and the estimated flux obtained by the discrete flux observer (3) in low speed operation. Fig. 1 also shows the real current and the estimated current. In Fig. $1(\mathrm{a})$, where the switching frequency is $8 \mathrm{kHz}$, the estimated flux is similar to the real flux. As the switching frequency decreases to $2 \mathrm{kHz}$, the estimated current becomes slightly different from the real current as shown in Fig. 1(b). However, the estimated flux is still close to the real flux.

If the motor speed increases to a medium speed range near the rated value, the estimated flux is a little bit different from the real flux, as shown in Fig. 2(a), even though the switching frequency is $8 \mathrm{kHz}$. If the switching frequency reduces to 2 $\mathrm{kHz}$ at this speed, the difference between the estimated flux and the real flux becomes significant, as shown in Fig. 2(b). Similar to the flux estimation, the error between the estimated current and the actual current becomes larger as the motor speed increases and the switching frequency decreases.

It can be concluded from the above results that, if the switching frequency isn't high enough, the simple discrete flux observer converted by the Euler method is not suitable for the medium speed operation of induction motors.
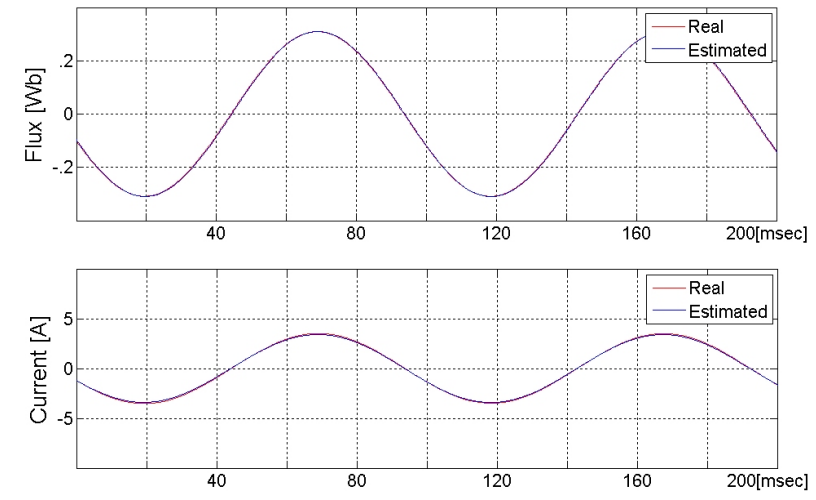

(a)
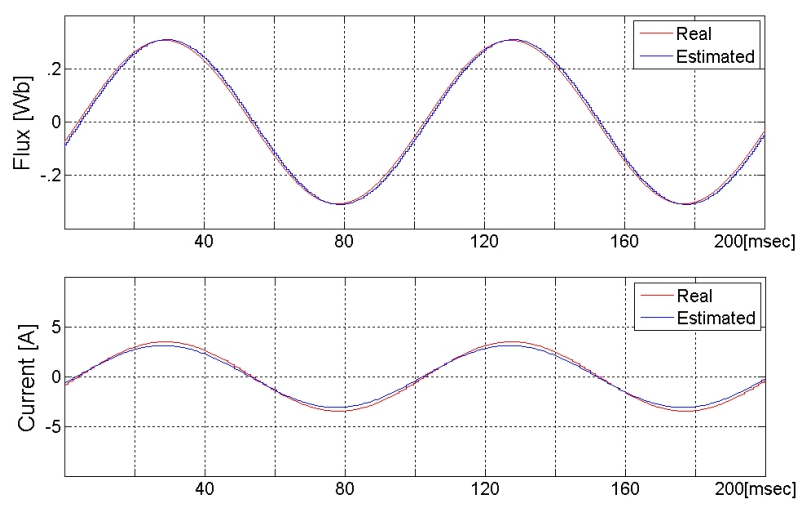

(b)

Fig. 1. The flux and the current estimated by the simple discrete flux observer at $10 \mathrm{~Hz}(300 \mathrm{rpm})$. (a) Sampling rate: $8 \mathrm{kHz}$. (b) Sampling rate: $2 \mathrm{kHz}$.

\section{The Power Series Approximation OF Discrete FLUX OBSERVERS}

Assuming that the motor speed $\omega$ is piecewise constant and the control input $\mathbf{u}$ is also constant during each sampling interval, the sampled system of (1) can be expressed as a linear difference equation [5].

$$
\begin{gathered}
\mathbf{x}(n+1)=\Phi \mathbf{x}(n)+\Gamma \mathbf{u}(n) \\
\Phi=e^{\mathbf{A} T_{s}} \\
\Gamma=\int_{0}^{T_{s}} e^{A \sigma} d \sigma \mathbf{B}
\end{gathered}
$$

The exact solution of (4) is to evaluate the matrix exponential in (5) and (6). Because $\Phi$ contains the time-varying component $\hat{\omega}, \Phi$ should be re-calculated at each sampling interval. As the calculation of the matrix exponential requires high computational load, (5) need to be simplified. One method for the evaluation of a matrix exponential is to use a power series expansion.

$$
\mathbf{e}^{\mathbf{A} T_{s}}=\mathbf{I}+\mathbf{A} T_{s}+\frac{\mathbf{A}^{2} T_{s}{ }^{2}}{2 !}+\frac{\mathbf{A}^{3} T_{s}{ }^{3}}{3 !}+\frac{\mathbf{A}^{4} T_{s}{ }^{4}}{4 !}+\cdots
$$

To reduce the computational load, a finite number of terms of (7) are taken for an approximation of the matrix exponential. 

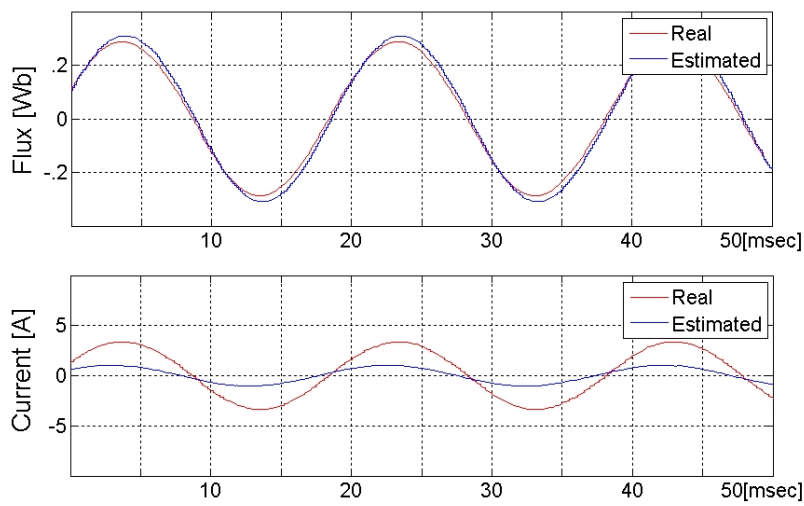

(a)
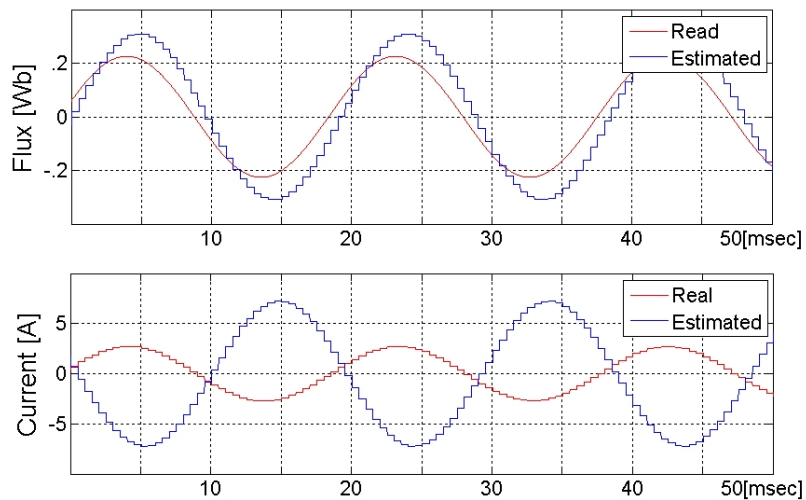

(b)

Fig. 2. The flux and the current estimated by the simple discrete flux observer at $50 \mathrm{~Hz}(1500 \mathrm{rpm})$. (a) Sampling rate: $8 \mathrm{kHz}$. (b) Sampling rate: $2 \mathrm{kHz}$.

The 1st order approximation takes the first two terms of (7). The result is the same as (3), which is converted by the Euler method.

$$
\mathbf{x}(n+1)=\mathbf{x}(n)+\mathbf{A} T_{S} \mathbf{x}(n)+\mathbf{B} T_{s} \mathbf{u}(n)
$$

In this paper, more terms are included during the approximation for the purpose of better precision.

The 2nd order approximation uses the first three terms of (7) for the calculation of $\Phi$ and $\Gamma$.

$\mathbf{x}(n+1)=\mathbf{x}(n)+\mathbf{A} T_{s} \mathbf{x}(n)+\frac{\mathbf{A}^{2} T_{s}^{2}}{2} \mathbf{x}(n)+\mathbf{B} T_{s} \mathbf{u}(n)+\frac{\mathbf{A} \mathbf{B} T_{s}^{2}}{2} \mathbf{u}(n)$

(9) can be rearranged as follows.

$$
\begin{gathered}
\mathbf{x}(n+1)=\mathbf{x}(n)+\mathbf{A} T_{s}\left[\frac{\mathbf{x}(n)}{2}+\frac{\mathbf{x}^{* 1}(n+1)}{2}\right]+\mathbf{B} T_{s} \mathbf{u}(n) \\
\mathbf{x}^{* 1}(n+1)=\mathbf{x}(n)+\mathbf{A} T_{s} \mathbf{x}(n)+\mathbf{B} T_{s} \mathbf{u}(n)
\end{gathered}
$$

(11) is the same equation as (8). The second term of (10) is the weighted sum of the present state and the next state obtained by first order approximation.

The 3rd order approximation uses the first four terms of (7) for the calculation of $\Phi$ and $\Gamma$. The 3rd order approximation requires one more computation of the state equation.
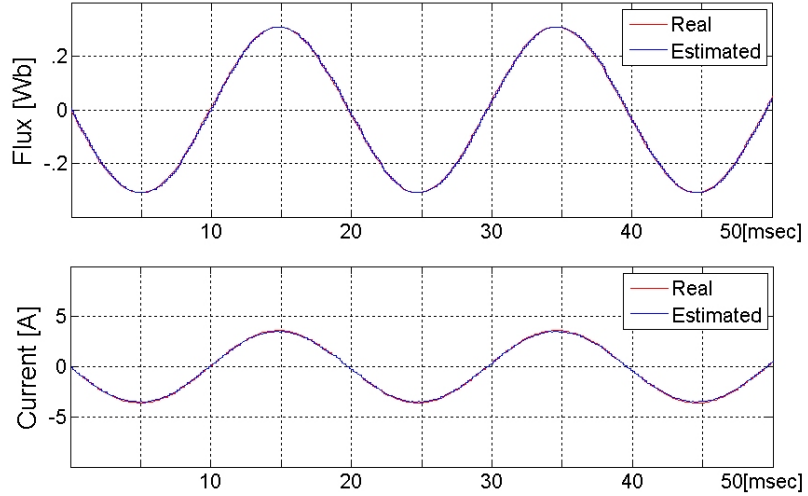

(a)
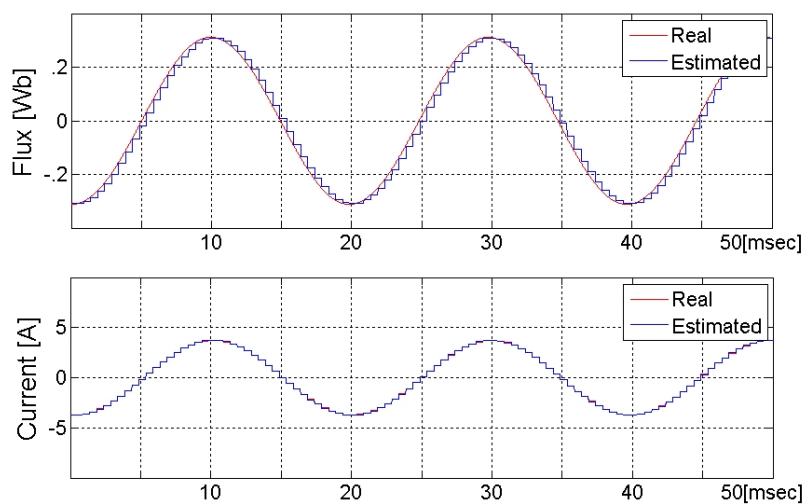

(b)

Fig. 3. The flux and the current estimated by the discrete observer with a 2nd order approximation at $50 \mathrm{~Hz}(1500 \mathrm{rpm})$. (a) Sampling rate: $8 \mathrm{kHz}$. (b) Sampling rate: $2 \mathrm{kHz}$.

$$
\begin{aligned}
\mathbf{x}(n+1) & =\mathbf{x}(n)+\mathbf{A} T_{s} \mathbf{x}(n)+\frac{\mathbf{A}^{2} T_{s}{ }^{2}}{2} \mathbf{x}(n)+\frac{\mathbf{A}^{3} T_{s}^{3}}{6} \mathbf{x}(n) \\
& +\mathbf{B} T_{s} \mathbf{u}(n)+\frac{\mathbf{A} \mathbf{B} T_{s}{ }^{2}}{2} \mathbf{u}(n)+\frac{\mathbf{A}^{2} \mathbf{B} T_{s}{ }^{3}}{6} \mathbf{u}(n)
\end{aligned}
$$

(12) can be rearranged as follows.

$$
\begin{aligned}
& \mathbf{x}(n+1)=\mathbf{x}(n)+\mathbf{A} T_{s}\left[\frac{\mathbf{x}(n)}{2}+\frac{\mathbf{x}^{* 2}(n+1)}{2}\right]+\mathbf{B} T_{s} \mathbf{u}(n) \\
& \mathbf{x}^{* 2}(n+1)=\mathbf{x}(n)+\mathbf{A} T_{s}\left[\frac{2}{3} \mathbf{x}(n)+\frac{1}{3} \mathbf{x}^{* 3}(n+1)\right]+\mathbf{B} T_{s} \mathbf{u}(n)
\end{aligned}
$$

$$
\mathbf{x}^{* 3}(n+1)=\mathbf{x}(n)+\mathbf{A} T_{s} \mathbf{x}(n)+\mathbf{B} T_{s} \mathbf{u}(n)
$$

The second term of (14) is the weighted sum of the present state $\mathbf{x}(n)$ and the next state $\mathbf{x}^{* 3}(n+1)$ obtained by (15).

The procedure of the 4th order approximation is similar to that of the 3rd order approximation, except for adding one more calculation of the state equation.

As the order of approximation increases for better precision, the calculation of the state equation also increases with the cost of more calculation time. The structure of the state equation in every calculation step is the same as in (8) except that $\mathbf{x}(n)$ in 

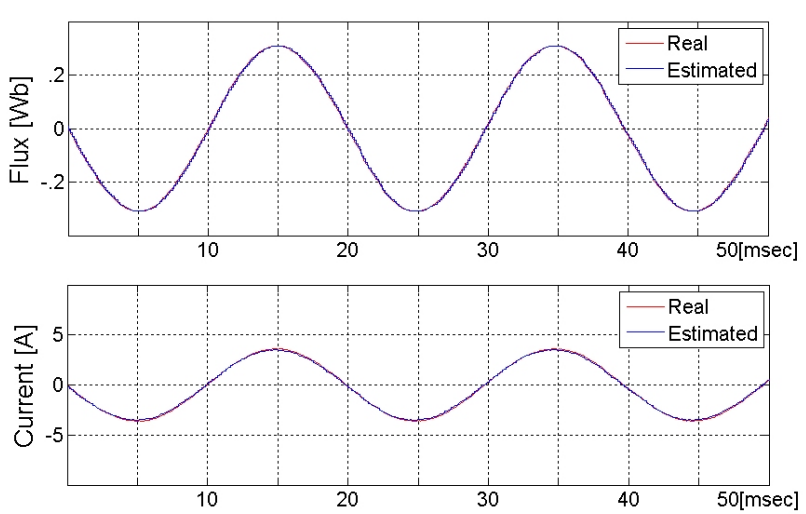

(a)
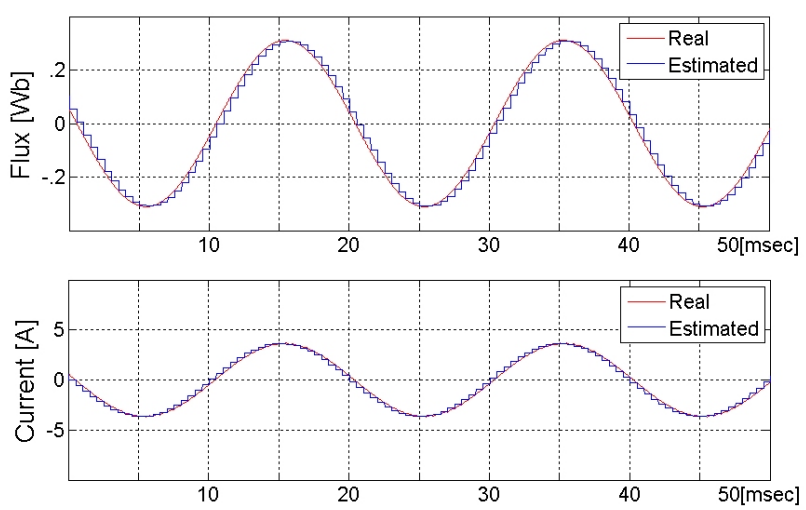

(b)

Fig. 4. The flux and the current estimated by the discrete observer with a $3 \mathrm{rd}$ order approximation at $50 \mathrm{~Hz}(1500 \mathrm{rpm})$. (a) Sampling rate: $8 \mathrm{kHz}$. (b) Sampling rate: $2 \mathrm{kHz}$.

the second term of state equation (8) is replaced by weighted sums of the present state and the future state. Therefore the same equation can be used repeatedly during the evaluation of state equations, which makes the programming relatively simple.

\section{Simulation II : Discrete Flux Observers with THE POWER SERIES APPROXIMATION}

In Fig. 3 and Fig. 4, the actual flux values are compared with the estimated flux values of two discrete flux observers which are converted through the 2nd and 3rd order approximations, respectively. The speed of the target motor is $1,500 \mathrm{rpm}$ at 50 $\mathrm{Hz}$ and the sampling rate of the control loop is $8 \mathrm{kHz}$ and 2 $\mathrm{kHz}$, which are the same conditions as in Fig. 2. Compared to Fig. 2, the flux observers with the power series approximation estimate the real flux with high precision. Observing Fig. 3 and Fig. 4, the power series approximation improves the performance of the discrete flux observer remarkably. It is also noted that, even if the order of approximation is increased from 2nd order to 3 rd order, there are no significant improvements in the medium speed range.

Fig 5 shows the performance of the discrete flux observer with the 2 nd order approximation at low speed operation. The flux observer estimates the actual flux precisely as expected.
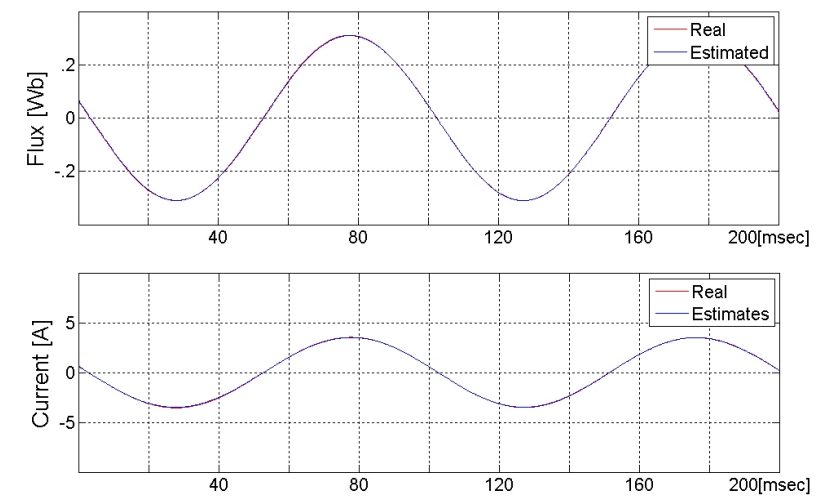

(a)
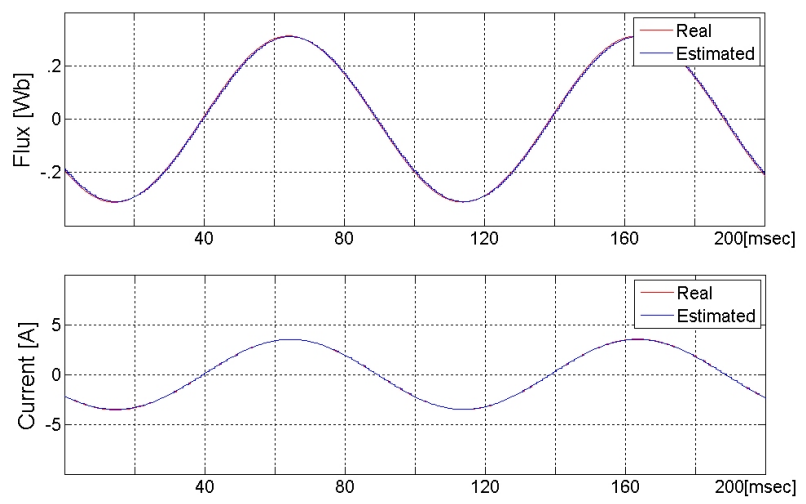

(b)

Fig. 5. The flux and the current estimated by the discrete observer with a 2nd order approximation at $10 \mathrm{~Hz}(300 \mathrm{rpm})$. (a) Sampling rate: $8 \mathrm{kHz}$. (b) Sampling rate: $2 \mathrm{kHz}$.

As the motor speed increases to a higher speed range with low sampling frequency, the 2nd order approximation is no longer enough for a precise estimation of the real flux. Fig. 6 through to Fig. 8 show the improvement of the estimation accuracy when the order of approximation is increased. The motor speed is $4500 \mathrm{rpm}$ with a sampling frequency of $2 \mathrm{kHz}$ and $8 \mathrm{kHz}$. When the sampling frequency is $8 \mathrm{kHz}$, all three methods estimate the real flux precisely.

However, if the sampling frequency is reduced to $2 \mathrm{kHz}$, the performance of the 2nd order approximation becomes inferior to that of the $3 \mathrm{rd}$ and 4 th order approximations, and the performance of the 4th order approximation is better than that of the 3rd order approximation.

Observing the simulation results, the 2nd order approximation has enough precision near the rated speed range. However, when the motor speed increases to a higher speed range, the order of approximation needs to be increased for precise estimation of the real flux.

\section{EXPERIMENT}

The validity of the simulation results is verified through an experiment. The target motor of the experimental system is similar to that of the simulation. As the real flux is not measurable, only the real current is compared with the estimated current. Because of the computational load, only 


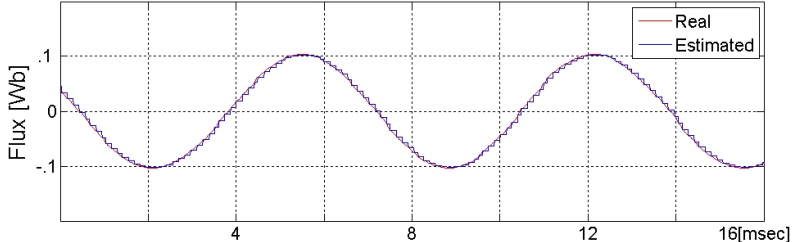

(a)

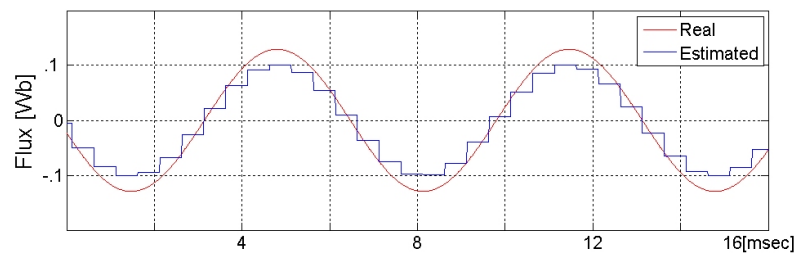

(b)

Fig. 6. The flux estimated by the discrete observer with a 2 nd order approximation at $150 \mathrm{~Hz}(4500 \mathrm{rpm})$. (a) Sampling rate: $8 \mathrm{kHz}$. (b) Sampling rate: $2 \mathrm{kHz}$.

the simple discrete observer and the observer with a 2 nd order approximation are implemented on the DSP.

Fig. 9 and Fig. 10 show the performance of the simple discrete observer and the discrete observer with a 2 nd order approximation at low speed operation. With the sampling frequency of $8 \mathrm{kHz}$, the estimated current coincides with the real current in both cases. If the sampling frequency is reduced to $2 \mathrm{kHz}$, however, the estimated current with the simple discrete observer becomes somewhat different from the real current as shown in Fig. 9(b), which is similar to the simulation result of Fig. 1.

The experimental results of Fig. 11 and Fig. 12 verify the simulation results of Fig. 2 and Fig. 3, respectively.

In the case of medium speed operation, the observer with a 2nd order approximation estimates the real current precisely with the sampling frequency of $2 \mathrm{kHz}$ and $8 \mathrm{kHz}$, as shown in Fig. 12. On the other hand, the difference between the real current and the current estimated by the simple observer is large even with the $8 \mathrm{kHz}$ sampling frequency, as shown in Fig. 11(a). The difference becomes larger if the sampling frequency reduces to $2 \mathrm{kHz}$, as shown in Fig. 11(b).

Through the experiment, the validity of Simulation I and Simulation II is confirmed, even though the estimated flux cannot be compared with the real flux.

\section{CONCLUSION}

From the simulation results and experimental results, it can be concluded that the power series approximation is an effective method for improving the accuracy of the discrete flux observer for medium and high speed drive of induction motors. Even though the order of approximation increases higher than the 2nd order, the performance improvement of the discrete flux observer is insignificant in the medium speed range. In contrast, in the high speed range, high order approximations such as 3rd order or 4th order approximation are more precise than the 2 nd order approximation.

Considering the accuracy and the computational load, the power series approximation with the 2 nd order approximation

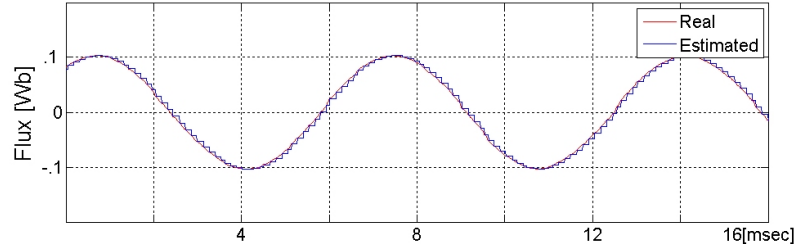

(a)

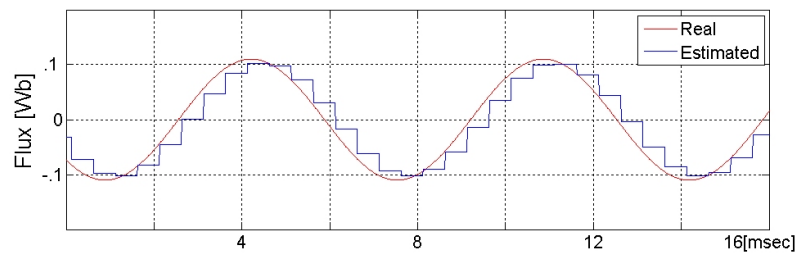

(b)

Fig. 7. The flux estimated by the discrete observer with a 3rd order approximation at $150 \mathrm{~Hz}(4500 \mathrm{rpm})$. (a) Sampling rate: $8 \mathrm{kHz}$. (b) Sampling rate: $2 \mathrm{kHz}$.

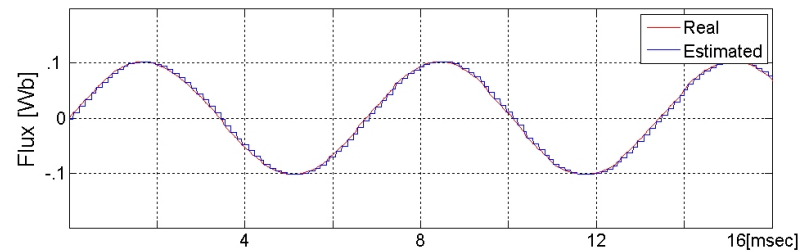

(a)

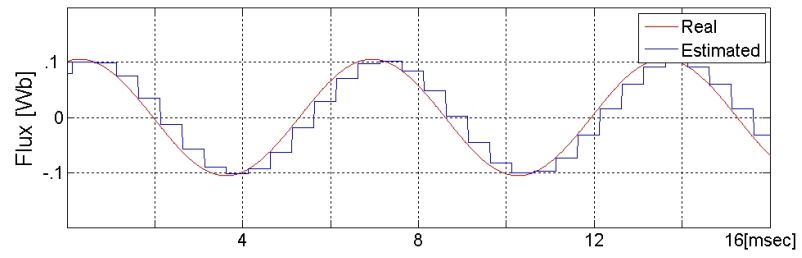

(b)

Fig. 8. The flux estimated by the discrete observer with a 4th order approximation at $150 \mathrm{~Hz}(4500 \mathrm{rpm})$. (a) Sampling rate: $8 \mathrm{kHz}$. (b) Sampling rate: $2 \mathrm{kHz}$.

is suitable for the flux observer design of general purpose motor drives. On the other hand, if high speed operation is required such as for machine tool applications, higher order approximations are adequate at the cost of high computational load. 


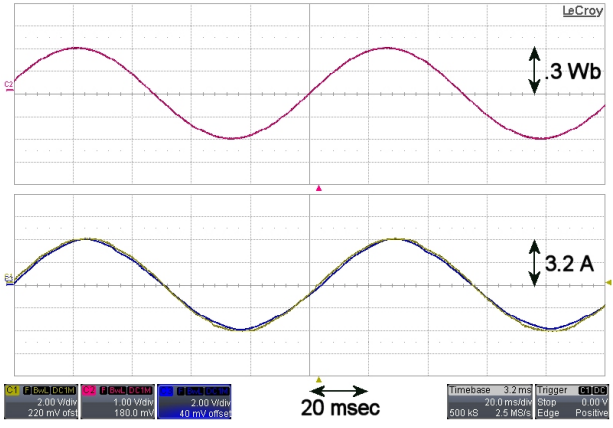

(a)

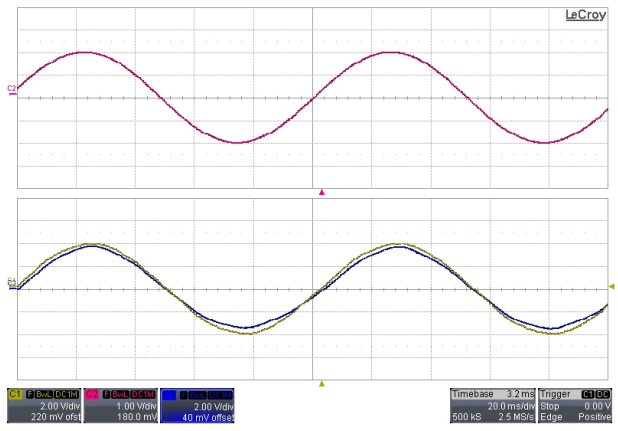

(b)

Fig. 9. The real current(yellow), the estimated current(blue) and the estimated flux(red) estimated by the simple discrete flux observer at $10 \mathrm{~Hz}(300 \mathrm{rpm})$. (a) Sampling rate: $8 \mathrm{kHz}$. (b) Sampling rate: $2 \mathrm{kHz}$.

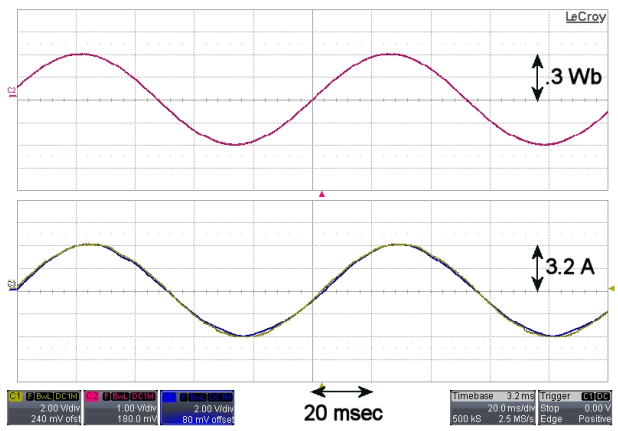

(a)

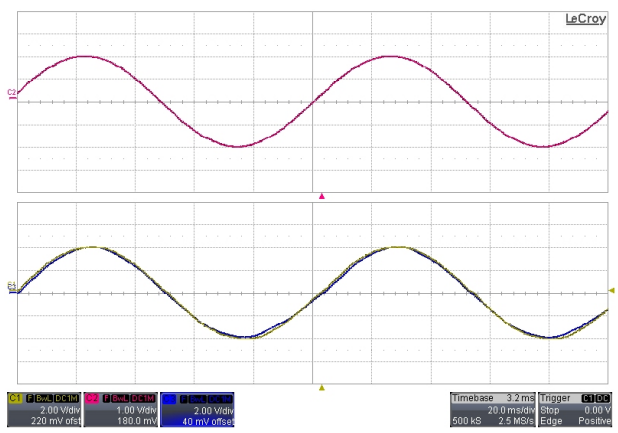

(b)

Fig. 10. The real current(yellow), the estimated current(blue) and the estimated flux(red) estimated by the discrete observer with a 2nd order approximation at $10 \mathrm{~Hz}(300 \mathrm{rpm})$. (a) Sampling rate: $8 \mathrm{kHz}$. (b) Sampling rate: $2 \mathrm{kHz}$.

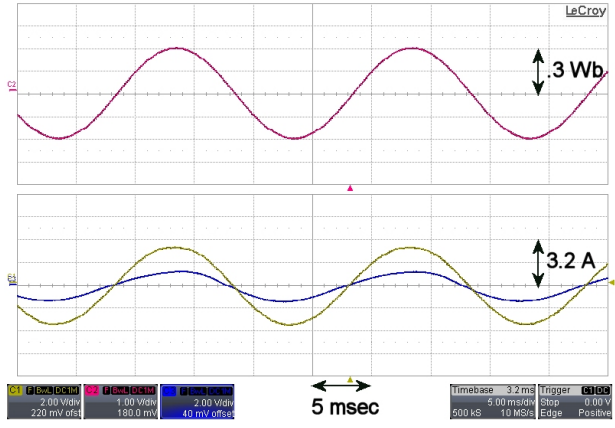

(a)

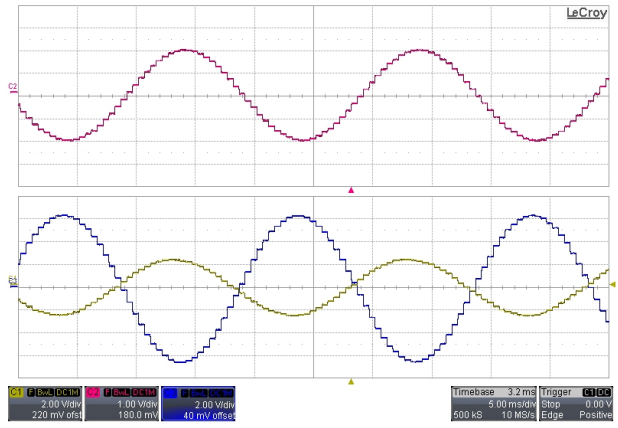

(b)

Fig. 11. The real current(yellow), the estimated current(blue) and the estimated flux(red) estimated by the simple discrete flux observer at $50 \mathrm{~Hz}(1500 \mathrm{rpm})$. (a) Sampling rate: $8 \mathrm{kHz}$. (b) Sampling rate: $2 \mathrm{kHz}$.

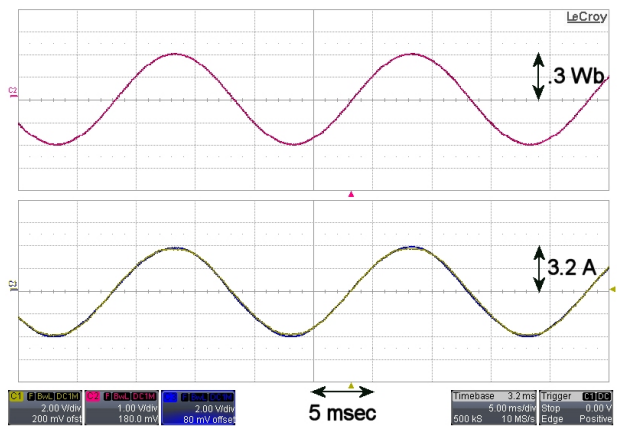

(a)

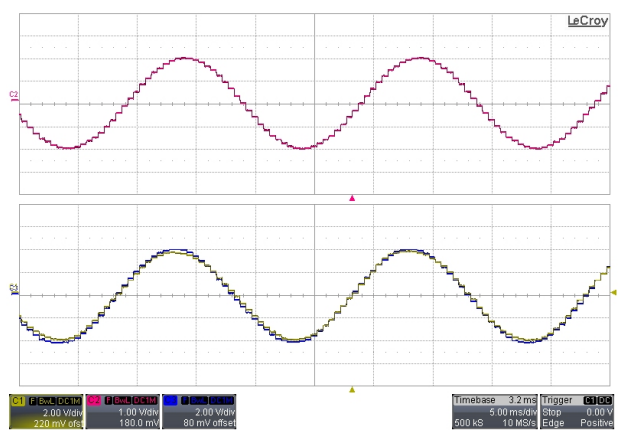

(b)

Fig. 12. The real current(yellow), the estimated current(blue) and the estimated flux(red) estimated by the discrete observer with a 2 nd order approximation at $50 \mathrm{~Hz}(1500 \mathrm{rpm})$. (a) Sampling rate: $8 \mathrm{kHz}$. (b) Sampling rate: $2 \mathrm{kHz}$. 


\section{REFERENCES}

[1] H. Kubota, K. Matsuse and T. Nakano, "DSP-based speed adaptive flux observer of induction motor," IEEE Trans. Ind. Appl., Vol. 29, No. 2, pp. 344-348, Mar./Apr. 1993.

[2] L. Harnefors and M. Hinkkanen, "Complete stability of reduced-order and full-order observers for sensorless IM drives," IEEE Trans. Ind. Electron., Vol. 55, No. 3, pp. 1319-1329, Mar. 2008.

[3] S. Suwankawin and S. Sangwongwanich, "Design strategy of an adaptive full-order observer for speed-sensorless induction-motor drives-tracking performance and stabilization," IEEE Trans. Ind. Electron., Vol. 53, No. 1, pp. 96-119, Feb. 2006.

[4] G. Foo and M. F. Rahman, "Wide Speed Direct Torque and Flux Controlled IPM Synchronous Motor Drive Using a Combined Adaptive Sliding Mode Observer and HF Signal Injection," Journal of Power Electronics, Vol. 9, No. 4, pp. 582-591, Jul. 2009.

[5] K. J. Astrom and B. Wittenmark, Computer Controlled Systems, PrenticeHall, chap. 3, 1984.

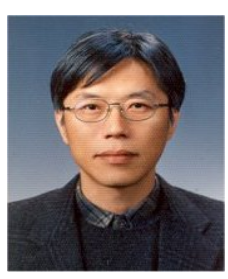

Kyung-Seo Kim was born in Seoul, Korea, in 1957. He received the B.S., M.S. and Ph.D. degrees in electrical engineering from Seoul National University, Seoul, Korea in 1982, 1984 and 1990, respectively. Since 1984 he has been with the LSIS R\&D Center, where he is currently a research fellow and the head of the Open Innovation Center. His research interests are in the areas of static converter for renewable energy, AC motor drives for electric vehicle and industrial applications.

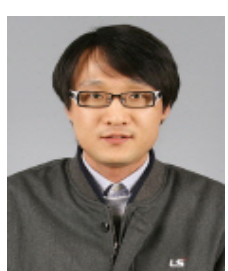

Il-Han Kim received the B.S. and M.S. degrees in electrical engineering from the Hanyang University in 2001 and 2003 respectively. From 2003 to 2011, he has been with the LSIS R\&D Center as a senior researcher $\mathrm{He}$ is currently a senior researcher at the Hyundai-Mobis R\&D Center. His research interests are in the areas of electric vehicle control and advanced control of AC machines. 